\title{
POSSIBILITÉS HYDROÉLECTRIQUES DU BASSIN HYDROGRAPHIQUE DU TAGE
} CONFÉRENCE FAITE A LA "CASA DU RIBATEJO" AU $11^{\circ}$ CONGRĖS DU RIBATEJO

\author{
José Filipe REBELO PINTO \\ Ingénieur-chef du Service d'Aménagements Hydroélectriques \\ au Ministère des Travaux Publics du Portugal.
}

\section{INTRODUCTION}

La Casa du Ribatejo m'a fait l'honneur de me demander de présenter à ce Congrès quelque; notes sur les possibilités hydroćlectriques du Tage; j'ai accepté cette invitation pensant que, malgré leur peu de mérites, ces notes seraient de quelque intérêt pour les habitants de la région à qui cette rivière donne la vie.

Le manque de données complètes, surtout en ce qui concerne la partie espagnole du bassin de ce fleuve, ne permet pas de donner une idce exacte de son importance du point de vue hydroélectrique. Avec les éléments que j’ai pu réunir. dans le court espace de temps dont j'ai disposé, j'ai aligné quelques notes ou, faute de mieux, je décris sommairement le bassin du Tage du point de vue hydroélectrique et je cite les installations les plus importantes - existantes, en construction et en projet -- tant au Portugal qu'en Espagne, indiquant, à l'approximation des données obtenues, leurs principales caractéristiques.

\section{LE BASSIN HYDROGRAPHIQUE DU TAGE DU POINT DE VUE HYDROELECTRIQUE}

Le bassin hydrographique du Tage (fig. I et VI) peut jouer un rôle important dans la Péninsule Ibérique et constitue un facteur économique assez puissant pour l'ensemble de la Péninsule, tant par l'extension des terrains susceptibles d'ètre irrigués par ses eaux, que par la grande quantité d'énergie que ces mêmes eaux peuvent fournir on encore par les possibilités de navigation quofrent le cours inférieur du Tage et son alfluent Sorraia.

Le Tage, sur un cours de plus de $1.000 \mathrm{~km}$, draine $55.800 \mathrm{~km}^{2}$ de terres en Espagne d $24.860 \mathrm{~km}^{2}$ au Portugal, au total 80.660 , soit une surface presque égale à celle du territoire portugais $\left(89.100 \mathrm{~km}^{2}\right)$. Il prend sa source dans les monts d'Albarracin, à une altitude de $1.593 \mathrm{~m}$. et sa descente jusqu’à la mer s'efrectue comme indiqué sur le profil en long schématique représenté sur la fig. II ; il descend de près de $800 \mathrm{~m}$. en un peu moins de $400 \mathrm{~km}$. et lorsqu'il passe a Tolède, il est dejà descendu de $1.150 \mathrm{~m}$.; lorsqu'il touche la terre portugaise (confluent de l'Erges) il lui reste seulement $98 \mathrm{~m}$. de dénivellés et moins de $300 \mathrm{~km}$. de parcours jusqu'à la mer. Le profil en long de son cours au Portugal et dans la zone frontiere (fig. III) montre que fo Tage s'abaisse de $20 \mathrm{~m}$. dans les $56 \mathrm{~km}$. du troncon frontière, compris entre les confluents de l'Erges et du Sever, $39 \mathrm{~m}$. dans les $54 \mathrm{~km}$. suivants (jusqu’à Belver), ensuile $30 \mathrm{~m}$. en $100 \mathrm{~km}$. (jusqu'à Porto Muge); et dans les 80 derniers km. d'un mètre seulement.

Il y aurait grand intérèt à présenter les profils en long des affluents les plus importants paree qu'ils constituent une donnée fondamentale pour évaluer les possibilités hydroélectriques. Il est impossible de le faire ici parce que cela exigerait un trop grand nombre de dessins et des descriptions tris longues. On peut cependant se faire une idée des dénivellées existantes dan; les diverses lignes d'eau en observant la fig. IV dans laquelle sont indiquées les chaînes de montagnes les plus importantes et les altiludes en différents points du bassin. On voit que le bassin supérieur du Tage et de ses principaux affluents de rive droite est situé dans des régions montagneuses de grande altitude - monts de l'Albarracin et Ibériques, Sierras de Guadarrama, Gredos, Gata et Estrella - et que leurs cours présentent de grandes dénivellées. Les aflluents de rive gauche naissent et coulent dans des régions moins élevées et par conséquent présentent des dénivellées plus faibles.

La carte udométrique de la Péninsule Ibérique (fis. I) donne une idée approximative de la distribution des pluies dans les divers bassins hydrographiques. On roit que les ressoures hydrauliques du Tage sont relativement modes- 
Les comparées a celles des rivières du Nord-Oues! de la Péninsule mais plus abondantes que celles d'autres situées à l'Est et au sud (par exemple, le Guadiana).

Dans la plus grande partie du bassin de rive gauche du Tage, les précipitations moyennes annuelles sont comprises entre 500 et $750 \mathrm{~mm}$. On enregistre dans certaines régions de faible étendue, plus à l'Est, des précipitations comprises entre 300 et $500 \mathrm{~mm}$; dans la plus grande partie du bassin de rive droite les précipitations moyennes anmuelles sont plus élevées puisque l'on enregistre des valeurs qui vont de 750 jusqu'à plus de $1.500 \mathrm{~mm}$. On observe les plus grandes précipitations dans la Serra da Estrella; viennent ensuite celles enregistrées dans les

\section{Bassins Hydrographiques de la Péninsule Ibérique.}

Carte Udométrique

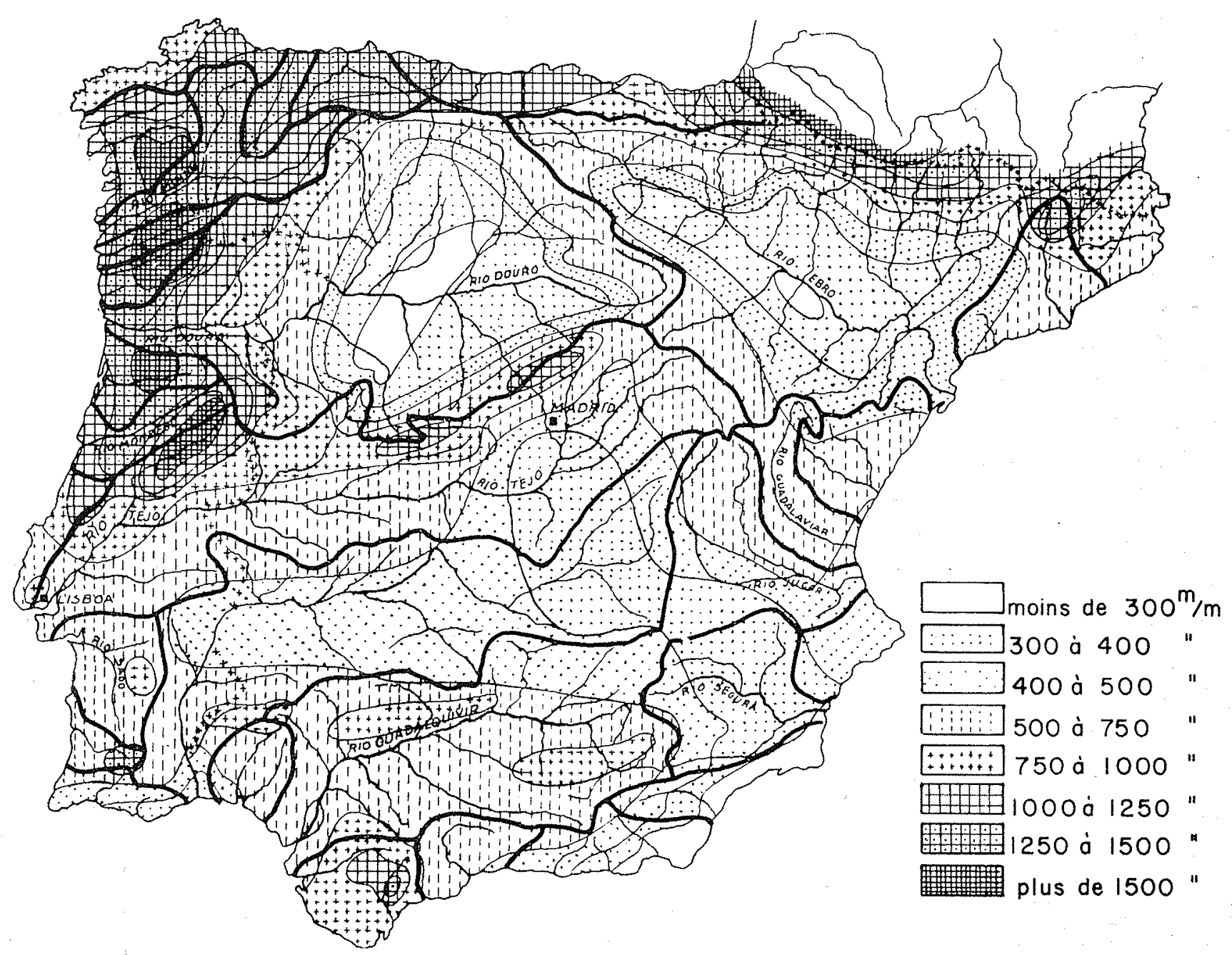




\section{Tage}

PROFIL EN LONG GENERAL DE L'EMBOUCHURE A LA SOURGE

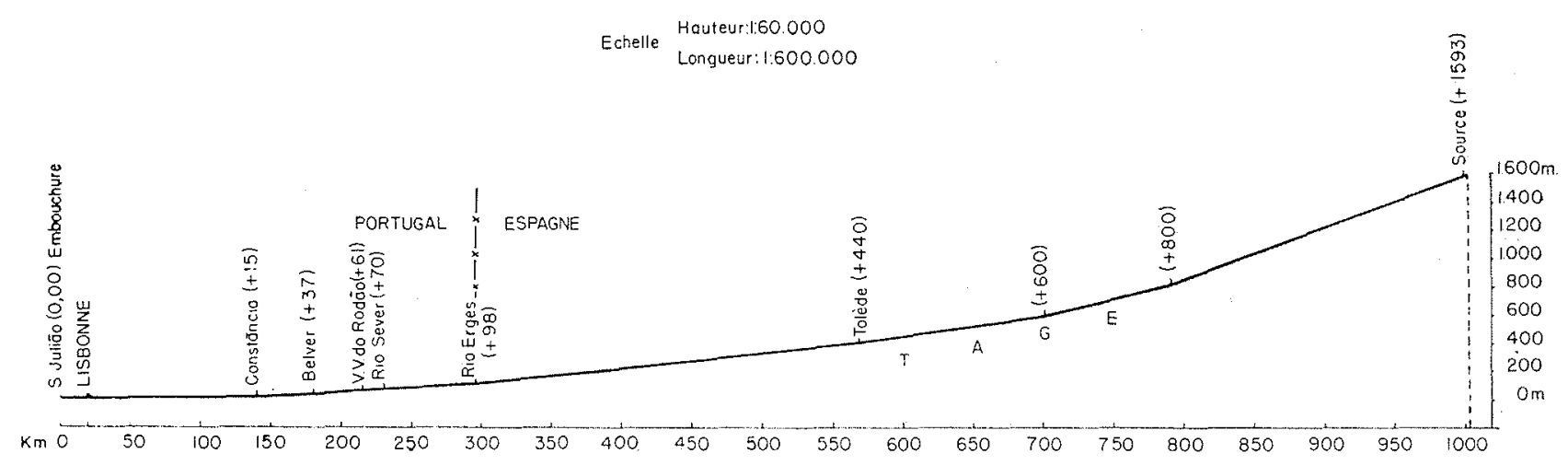

Sierras de Gredos et de Guadarama; dans ces zones les précipitations soas forme de neige prennent une certaine importance.

Les pluies sont tros irrégulières, variant tant en quantité annuelle qu'en distribution au cours de l'anné ; on observe par conséquent une grande irrégularité des débits du fleuve. Sur la fig. IV sont représentés les débits moyens anntels observés à Rodào et à Bolarque (Espagne) depuis 1913 jusquà 1945. Ces graphiques montrent la grande variation des débits d'une année à l'autre; cette variation est plus accentuce a Rodào, où la surface du bassin versant s'élève à $59.167 \mathrm{~km}$, qu'à Bolarque où la surface du bassin versant n'est que de $7.600 \mathrm{~km}$. A Rodào on observe une variation d'un minimum de $200 \mathrm{~m} / \mathrm{sec}$. (en 1944) à un maximum de plus de $1.000 \mathrm{~m}^{3} / \mathrm{sec}$. (1936). A Bolarque, les valeurs maximum el minimum sont respectivement de $102 \mathrm{~m} 3 / \mathrm{sec}$. (en 1941 ) et 18 en 1945. L’irrégularité des débits est encore mise en relief par les graphiques de la fig. $V$ où sont indiqués les débits moyens mensuels de cing années successives - 1934 à 1938.

Encore plus flagrante est la différence entre les débits instantanés des différentes époques de l'année - à Rodào on a enregistré un débit maximum de crue de $15.850 \mathrm{~m}^{3} / \mathrm{sec}$. le 7 décembre 1876 , et un minimum de quelques centaines de litres par seconde dans l'étiage exceptionnel de 1945, durant lequel on a observé des débits voisins ou inférieurs à $1 \mathrm{~m}^{3} / \mathrm{sec}$, dès le milieu de juillet jusqu'au milieu d'octobre. Ces nombres correspondent à des valeurs extrèmes rarement atteintes. On enregistre cependant toutes les années ou presque toutes les années des débits de crue de quelques milliers de $\mathrm{m}^{3}$ par seconde et des débits minimum d'étiage compris entre 10 et $20 \mathrm{~m}^{3} / \mathrm{sec}$.

Les périodes de hattes eaux et de basses eaux ne s'établissent pas à la mème époque chaque année; les hautes eaux s'observent normalement de Novembre à Arril ; il arrive parfois lorsqu'il y a coïncidence de pluies abondantes el de la fonte de masses importantes de neige dans les montagnes espagnoles, qu'on enregisire des débits élevés à des époques plus avancées de l'annéa. Comme exemple, on peut citer le mois de mai 1946 durant lequel on a enregistré a Rodào des débits supérieurs à $1.000 \mathrm{~m} 3 / \mathrm{sec}$. du $1^{\text {er }}$ a 15 de ce mois avee un débit maximum de $6.200 \mathrm{~m} 3 / \mathrm{sec}$ le 2 mai. L'étiage commence no:malement en mai ou juin et se prolonge jusqu'à octobre ou novembre, avec des débits minimum en aoùt ou septembre. La date du début et de la fin de l'étiage varie beaucoup parce qu'elle est liée tant à l'époque qu'à la natare des précipitations - pluies on neiges - et aussi à la température, laquelle influe sur la nature tes precipitations et aussi sur la fonte des neiges. Une fin d'hiver et un début de printemps très frais peavent retarder le début de l'étiage qui, au contraire, commencera plus tòt si cette époque de l'année est plus chaude. Une fin d'automne et un commencement d'hiver froids peuvent, allics à de faibles précipitations, prolonger l'ćtiage jus. qu'à décembre ou mème janvier.

Le regime des principaux affuents du Tago diffère un peu d'un affluent à l'autre et varie avec la situation du hassin versant; présentent normalement des étiages plus accentués et de plus longue durée, ceux situés dans des régions moins montagneuses, plus basses et de pluviosité plus faible. La plus grande partie des affuenis rive gauche et quelques-ums de ceux de rive droite sont, dans ces conditions. L'influence des neiges n'a d'importance que sur les affluents de rive droite, plus spécialement sur ceux qui prennent naissance dans les Sierras de Gredos et de Gua- 
dirrama. Des affluents portugais, seul le Zézere a des débits parfois influencés par la fonte des neiges de la Serra da Estrella.

\section{INSTALLATIONS HYDROELECTRIQUES}

L'énergie des eaux du bassin hydrognaphique du Tage est utilisée de longue date par des moulins et de pelites industries. Au début de ce siccle on a commencé à installer des centrales hydroélectriques destinces à satisfair? les exigences d'une consommation privée on de caractère local ; telles étaient celles; construites dans le but dalimenter les fabriques de papier élablies sur les rives du Nabao, les filatures et les tissages de laine situés sur les versants de la Serra da Estrella, el d'autres encore sur l'Almonda, l'Alviela et de petits sousaflluenis du Tage. Ces installations étaient généralement constituces d'abozd par de simples ouvrages de dérivation, presque toujours résultant de l'adaptation d'anciens barrages de tres faible hateur et de canaux parfois centenaires, qui avaient servi ordinarement pour alimenter des moulins et des roues, et ensuite par des centrale; de basse chute et de faible puissance.

De telles installations ne disposaient cependani pas d'énergie en été, faute d'eau; d'autres n'en disposaient pas non plus en période de hautes eaux, faute de chute ; les propriétaires étaient alors obligés de suspendre le travail, ou d'installer des centrales thermiques pour compenser ce manque d'énergie.

Les besoins toujour's croissants d'énergie, les difficultés d'approvisionnement en combustible pour alimenter les centrales thermiques et do coût élevé de ces productions (très peu d'heures ('utilisation) conduisirent a envisager une meilleure utilisation des rivieres.

On a commencé alors à fiaire des études plus complètes du régime des rivieres, installant dans ce but un grand nombre de postes d'observation munis d'appareils destinés à la mesure des pluies et des hauleurs d'eau des rivieres, et l'on a effectué des mesures systématiques de débit. Dans les bassins portugais du Tage se trouvent installés 92 postes udométriques et 4 udographi. ques, 27 cehelles hydrométriques et 11 postes limnigraphiques. De front avec ces études hydrologiques on a effectué des levés topographiques et poursuivi des études géologiques afin d'obtenir des renseignements certains sur les possibilités hydró́lectriques des rivières - disponibilités en eau et en chute et conditions techniquement et économiquement favorables à l'exécution des ouvages correspondants.

\section{Tage}

Profil en long du cours au Portugal et de la zone frontiere entre Espagne ef. Portugal

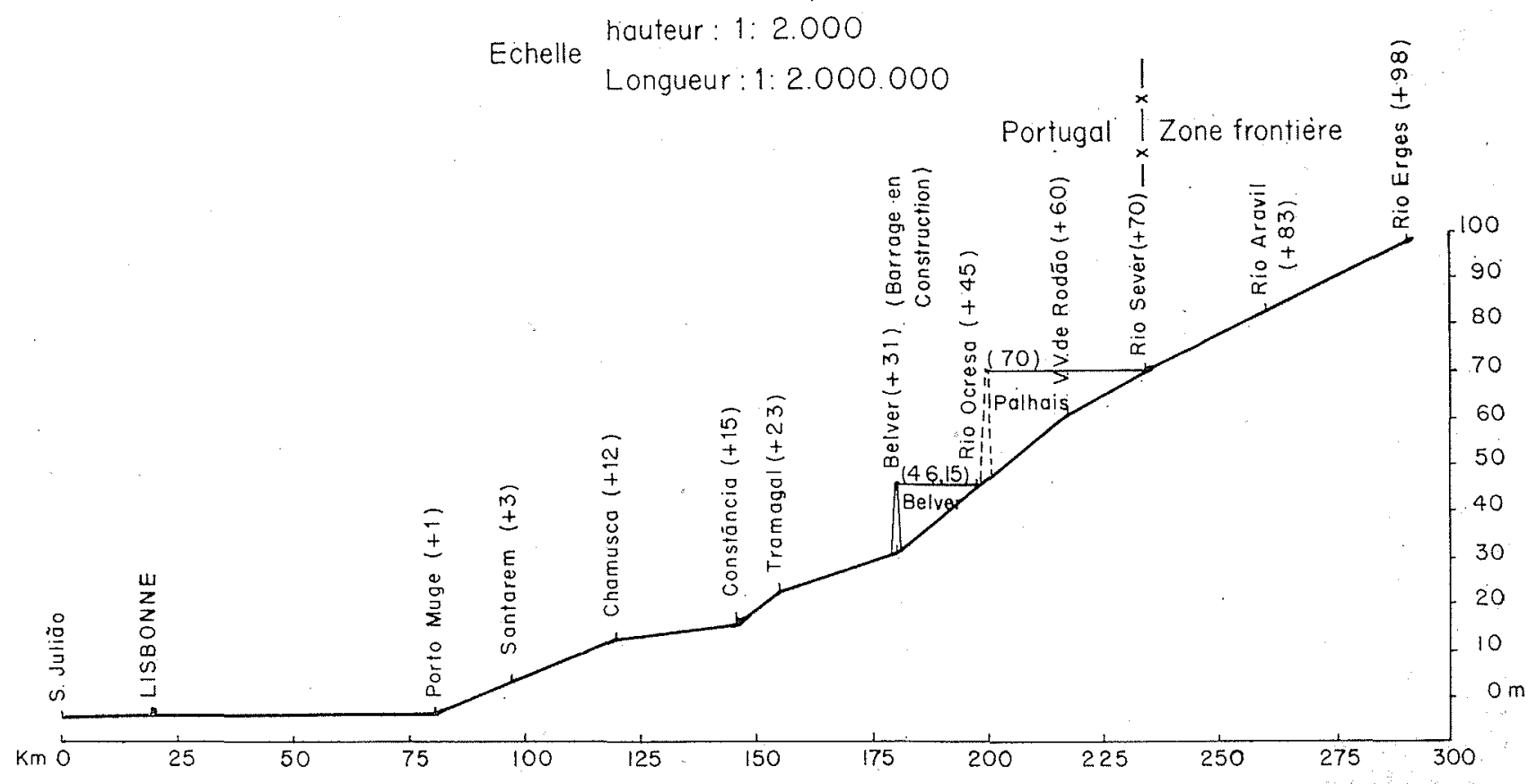


Ces études ont montré que pour mieux utiliser l'énergie des rivières, il était indispensable de créer de grandes retenues destinées à emmagasiner les eaux abondantes des périodes to

$$
\begin{aligned}
& \text { Débit moyen annuel du TAGE } \\
& \text {-... "àlarque (surface du bassin } 7.600 \mathrm{~km}^{2} \text { ) } \\
& \text { "Rodāo (" " }
\end{aligned}
$$

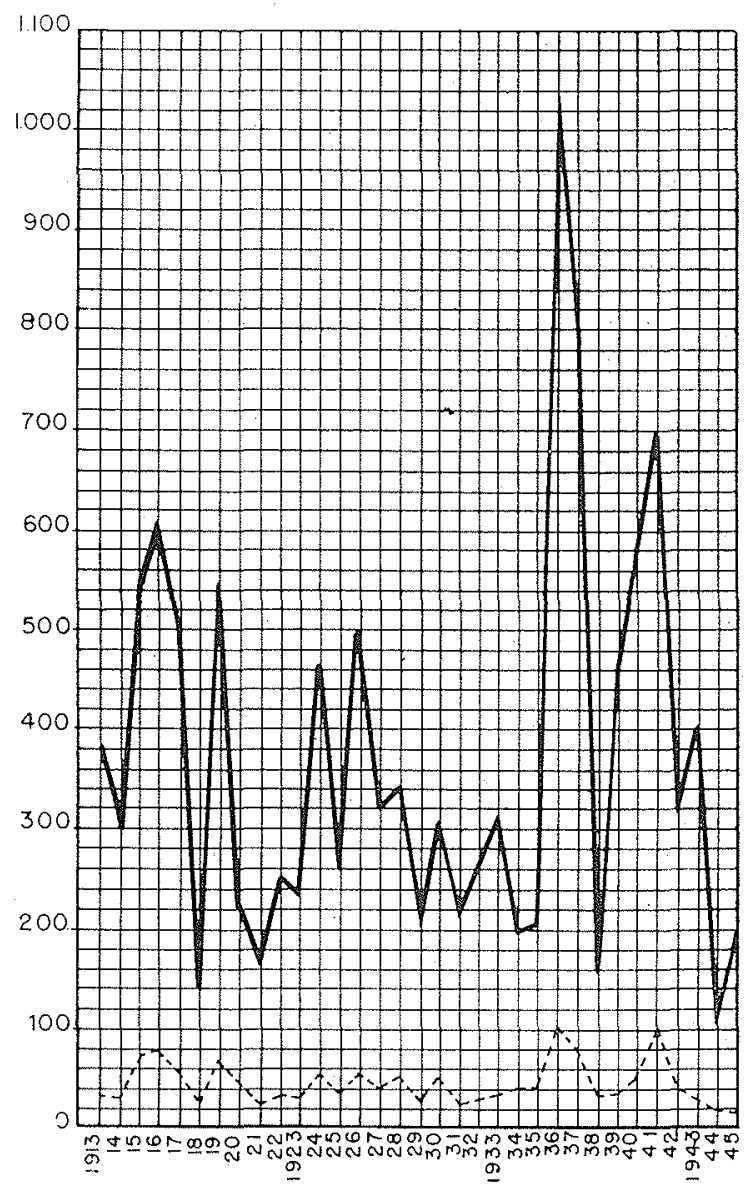

crues et de débits ćlevés et à les restituer peradant l'étiage.

Dans le bassin da Tage, pour permetre la régularisation annuelle du débit, éestà-dire pour obienir un débit constant pendant toute l'année, il faudrait créer des retenues d'une capacilé utile de l'ordre de la moitié du clébit intégral du fleuve. La valeur de la capacité nécessaire est cependant plus faible ou au contraire plus grande selon que la pluviosité dans les bassins est plus ou moins grande, et que la distribution des pluies au long de l'année est plus ou moins favorable.

Etant donnée la grande variation des débits intégraux annuels pour pouvoir utiliser toute l'eau qui passe dans les rivières, il faudrait des retenues de capacité énorme, capables d'emma- gasiner l'exces d'eau des années les plus humides pour les restituer atx rivières au cours des années seches re... renues de régularisation inter-annuelle.

Il n'est cependant pas possible, dans bien des cas, de créer des retenues d'une capacité suffisante pour permettre la régularisation annuelle des débits parce que les conditions topographiques ou géologiques des vallées ne le permeltent pas ou parce que ces retenues inonderaient des roies de communication importantes, de grandes agglomérations ou des surfaces très étendues d'excellentes terres cultivables, ce qui donnerai? lieu à des problèmes techniques, économiques ou sociaux, dont la résolution serait très difficile ou démesurément onéreuse. Les conditions de cette nature sont typiques dans la vallée du Tage - ligne de chemin de fer de Beira Baixa; nombreux ki'omètres de routes, quelques ponts, diverses agglomérations et d'importantes surfaces culivées à des cotes à peine supérieures a celles de la rivière.

Les difficultés soulevées par la création de rete. nues de régularisation annuelle des débits sont, ividemment, très aggravées pour les retenues de régularisation inter-annuelle. Il est très rare qu'on puisse rencontrer des conditions naturelles favorables à la création de retenues de ce type et mème quand cela arrive, les dépenses nécessitées par l'établissement d'une telle retenue se justifient difficilement du point de vue économique.

Il $\mathrm{y}$ a pourtant des cas spéciaux où se justifie la création de retenues de régularisation inter-

Débit du TAGE à Rodöo

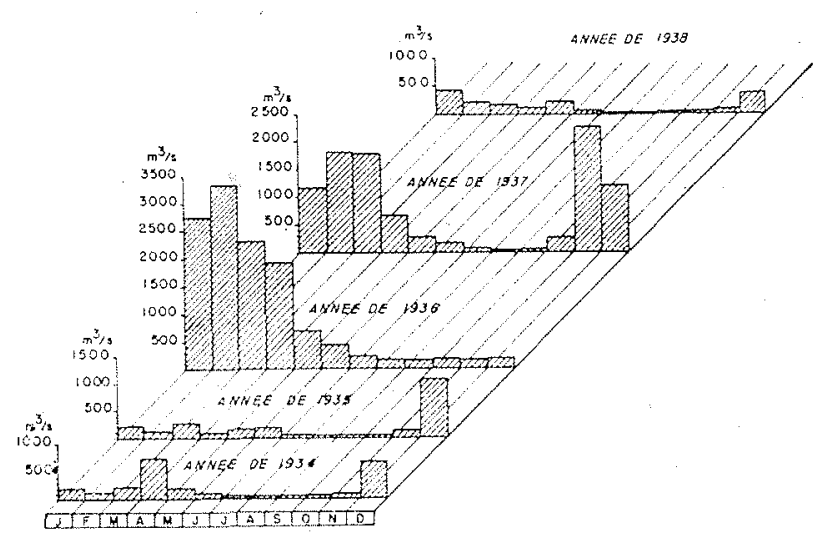

annuelle ; cela a été compris par nos voisins, comme on verra dans la suite.

Dans le bassin portugais du Tage, la première installation hydroélectrique disposant d'une retenue capable d'une régularisation appréciable des 
débits - la retenue de Povos sur la « ribeira" de Nisa, d'une capacité de 22.000 .000 de $m^{3}-$ est entrée en exploitation en 1927. Peu après, on créait immédiatement à l'aval, une autre retenue de 6.000 .000 de $\mathrm{m}^{3}$ de capacité, afin d'améliorer la régularisation obtenue par la première. En 1943, la retenue de Santa-Luzia, sur la Pampilhosa est entrée en service ; d'une capacité de 44.000 .000 de mis, elle sert à emmagasiner toute l'eau tombée dans le bassin correspondant pendant les mois de précipitations abondantes et à restituer cette eau à la centrale seulement au cours des époques d'étiage.

La retenue de Cabeco Monteiro d'une capacité de 78.000 .000 de $\mathrm{m}^{3}$, qui emmagasine les eaux da Ponsul, en vue d'alimenter les canaux d'irrigation de la Campine d'Janha et de produire accessoirement de l'énergie électrique, a été terminée en 1946.

La mème année, on a commencé les ouvrages de la chute de Pracana, dont la retenue emmagasinera 90.000 .000 de $m^{3}$ des eaux de l'Oereza ct les travaux préparatoires pour la cons?ruction de la grande centrale hydroélectrique de Casteio do Bode sur le Zézere où sera créé, par un barrage de $102 \mathrm{~m}$. de hauteur, une retenue de 1.070 .000 .000 de $\mathrm{m}^{3}$ de capacité totale et de 875.000 .000 de capacié utile.

Dans la partie espagnole du bassin du Tage, les choses se sont passées de façon analogue a celles décrites pour le Portugal, avec la seule différence que nos voisins ont commencé plus tôt et qu? les retenues construites ont été destinées initialement à des fins hydro-agricoles oil à l'alimentation en eau d'agglomérations importantes ef seulement quelques années après à la produclion d'énergie hydroélectrique. On citera comm exemple des premieres, les retenties du rio Lozoya, qui alimente en eau la ville de Madria et des secondes les retenues du rio Alberche.

Lorsqu'on eut obtenu plus de renseignements sur les besoins en eau pour l'irrigation el pour la production d'énergie et sur les ressources naturelles on a ébauché de vastes plans d'utilisation des eaux du bassin du Tage tant en Portugal qu'en Espagne. Ces plans ne sont pas encore complets et ne seront certainement pas établis d'une facon définitive dans les prochaines années. C'est que l'évolution des besoins des populations, de la technique et des conditions économiques ne permet pas d'élanorer des plans pour une exécution dans un futur éloigné ; il faul ajuster les besoins avee les possibilités maté. rielles et économiques et ce qui, aujourd'hui, ne parait ni techniquement ni ćconomiquement viable peut, dans quelques années, devenir d'une exécution facile ou présenter des conditions éco- nomiques très différentes ou encore ètre justifié par des nécessités sociales impératives.

Il nous a blé permis d'observer un exemple firgrant de cette assertion entre 1939 et maintenant; de nombreuses installations hydroélectriques qui présentaient alors des conditions économiques peu encourageantes deviennent aujourd'hui, par-suite de la hausse de prix des combustibles et, en particulier, des difficultés d'approvisionnement en charbon, d'exécution urgente et d'exploitalion assez favorible sous l'aspect financier.

Mais il est aussi possible que des plans élaborés aujourdhui deviennent désuets dans un court espace de temps. Peut-etre disposera-t-on, alors, de nouvelles sources d'énergie plus puissantes et plus économiques que celles connues jusqu’ici.

En ce qui concerne les installations hydroagricoles, les conditions peuvent aussi varier en peu de temps; une augmentation rapide de la population ou la simple disparition d'un fort courant migratoire, le manque ou l'abondance mondiaux de denrées alimentaires avec les hausses ou les baisses de prix qui s'ensuivent, des difficultés, facilités ou taxations du commerce international, ou la hausse du standing de vie des populations provoquée par la mise en valeur de produits naturels ou de l'industrie peuvent exiger une exploitation agricole plus intense qui ne peut etre obtenue que par l'irrigation ou, au contraire, conduire à ajourner la réalisation de plans dont l'étude est déjà terminée.

Pour ces raisons, les plans élaborés en Espagne, et dont on a connaissance, ne permettent pas de prévoir les changements qui viendraient atérer le régime des débits du Tage dans un futur: prochain ou lointain, changements qu'il serait intéressant de connaître pour utiliser au mieux ses eaux au Portugal.

Il y a, me semble-t-il, dans la partie espagnole de son bassin, près de 40.000 ha de terres qui peuvent être irriguées dont la moitié ou le tiers présente des conditions plus favorables poui lirrigation. Comme les besoins d'énergie en Espagne sont prédominants, la construction d'ouvages de régularisation pour des fins hydroelectriques est done urgente - et comme le passage de cultures en terrain see à celles en terrain irrigué se fait toujours lentement, il $y$ a lieu d'espérer que, dans un futur prochain, nous riendrons à bénéficier de cetle régularisation. Pour combien de temps ce bénéfice se maintiendra-t-il et à quel degré? Là est la question a laquelle on ne peut répondre. Tout dépend de l'extension qui sera donnée aux irrigations en Espagne, extension qui, pour sa part, est liée a tous les facteurs ci-dessus indiqués. 
Sur le tableau I el la lis. VI sont indiquées les installations hydroclectriques les plus importantes du bassin hydrographique du Tage existantes, en construction on en projet - en Espagne. Il est naturel qu'il y ait des omissions, que quelques-unes des installations indiquées no soient pas destimées à la production d'énergie on tout au moins que ce ne soit pas là leur principale fonction, et que les caractéristiques mentionnées ne correspondent pas bien à la réalité. Faute d'informations complètes, j’ai compilé des données d'origines différentes et qui n'étaient pas toujours concordantes, ce qui a certaine. ment donné lieu à quelques inexactitudes.

Des 48 installations indiquées au tableau I, onze sont terminces et en exploitation et dispom sent de retenues dont la capacité totale reprisente approximativement 330.000 .000 de $\mathrm{m}^{3}$ et produisent anmuellement près de 240.000 .000 de kwh; 8 sont en construction et auront des retenues d'une capacité utile totalisant $2.770 \mathrm{mil}$ lions de $\mathrm{m}^{3}$; elles sont destinées à produire quelque 260.000 .000 de $\mathrm{kwh}$; les 29 restantes sont encore en cours de projet ou d'étude et bien que les caractéristiques de quelques-unes d'entre alles ne soient pas encore comp'ètement définies, nous jugeons que leurs retenues emmagasineront près de 500.000 .000 de $\mathrm{m}^{3}$ et que leurs cestrales fourniront quelque 200.000 .000 de kwh.

Il n'eś pas facile de savoir quel effet régulateur auront les retenues espagnoles éant donnés les régimes d'exploitation très différents auxque's elles sont desinées. Les retenues construites par l'Etat Espagnol -... parmi lesquelles on pout citer celles de Entrepenas, Buendia, Vado, Rosarito, Gabriel y Galan et Borbollon -.. sont destinées à la régularisation ou la compensation des débits, les concessionnaires des centrales correspondantes (qui sont construites par eux) ayant l'obligation d'utiliser leurs eaux selon un régime qui leur est imposé. Celles d'Entrepenas (Tage) et de Buendia (Guadiela), reliées entre elles par une galerie, constituent un exemple typique de régularisation inter-annuelle, ainsi que le montre le rapport entre leur capacité et les débits intégraux moyens annuels -.. $750+1518=2268$ et $750-710=1460 \mathrm{mil}$. lions de $\mathrm{m}^{3}$; l'exploitation réunie de ces deux retenues permet d'inverser totalement le régime naturel des rivières qui s'y jettent et l'on prévoit que le débit qu'elles fourniront au 'Tage prendra des valeur's comprises entre un minimum de l'ordre de $20 \mathrm{~m} 3 / \mathrm{sec}$. en hiver et un matximum en été un peu supérieur à $80 \mathrm{~m}^{3} / \mathrm{sec}$. Ces valeurs sont sujettes à des rariations car l'exploitation de ces deux grandes retenues sera certainement orientée vers la compensation des dé- bits tant pour lirrigation de la vallée du Tage que vers la production d'énergie dans leur propre centrale et dans celles qui sont plus à l'aval elles devront fournir des débils plus petits dans les années humides, el plus ćlevés dans les années sèches.

La capacité des 4 autres retenues el le débit intégral moyen des bassins respectifs sont, en millions de m3 : Vado 38 et 213; Rosarito 67 et 665 ; Gabriel y Galan 234 et 557 ; Borbollon 75 et 113. Ces nombres montrent qu'en année moyenne on doit obtenir une régularisation complète des débits dans la retenue de Borbollon, une régularisation très élevée dans celle de Gabriel y Galan, et de faibles régularisations dans celles de Vado et de Rosarito.

Pour les retenues restantes, je n'ai pas pu obtenir de données complètes; leur exploitation sera naturellement conditionnée par les nécessités de l'irrigation ou de la production d'énergie, suivant le but auquel chacune est destinée, par la distribution des pluies et la variation des autres facteurs climatiques au cours de l'année et dans toute l'Espagne.

Un calcul approximatif de la régularisa'ion obtenue arec toutes les retenues indiquées au tableau I conduit à supposer qu'en année moyenne, les débits fournis en étiage doivent ètre de l'ordre de $160 \mathrm{~m} 3 / \mathrm{sec}$. dont 20 reviennent aux installa ions existantes, 110 à celles qui sont en construction et 30 à celles qui sont encore en cours de projet ou d'étude.

Il n'est pas facile d'évaluer le bénéfice résultant de la régularisation effectuée par les ouvrages espagnols sur le régime du Tage à son entrée au Portugal et, en particulier en ce qui concerne les débits d'étiage. Il y a cependant lieu d'espérer que dans les premières annees après entréc en service des retenues qui sont actuellement en construction, les débits d'étiage subiront une augmentation de quelques dizaines de $\mathrm{m}^{3}$ par secon. de. Avec l'intensification des irrigations en Espagne, ce bénéfice ira en diminuant, il se peut mème que dans un futur plus ou moins lointain. 'irrigation du pays voisin absorbe presque complètement le débit d'étiage du Tage.

On peut cependant compter sur un bénéfice certain en ce qui concerne la réduction du débit de: crues venues d'Espagne. Les retenues indiquées dont la capacité totale doit atteindre environ 3.600 millions de $\mathrm{m}^{3}$, dominent $14.000 \mathrm{~km}$, soit environ $1 / 4$ de la surface du bassin du Tage en Espagne. Si l'on tient compte du fait que les bassins dominés par les retenues les plus importantes sont situés dans les régions de plus grande pluviosité, on peut admettre que les débits at l'entrée de la frontière subiront une réduction 
TABLEAU I

Aménagements hydroélectriques dans le bassin hydrographique du Tage en Espagne.

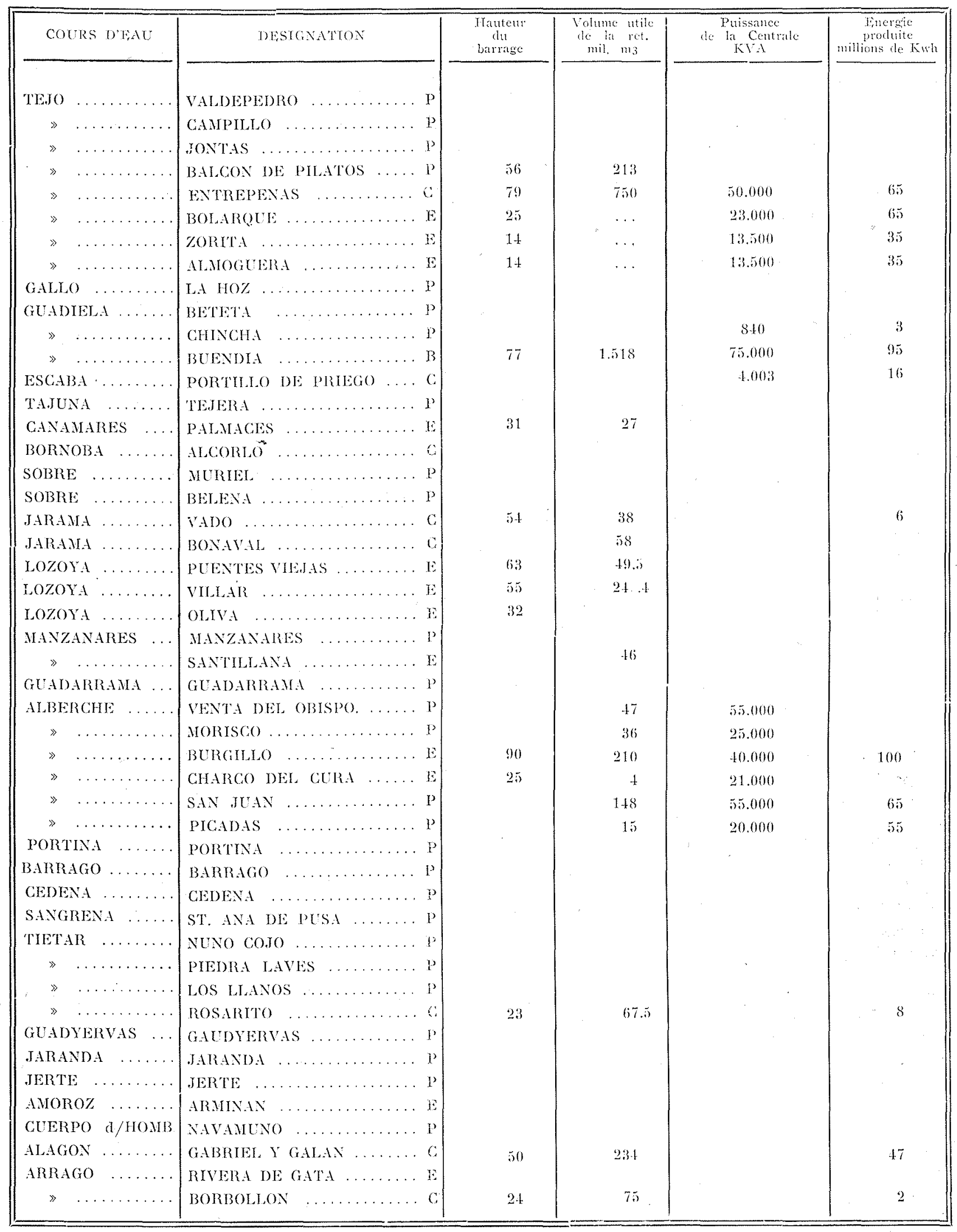

P : en projet - C : en construetion - E : en exploitation. 


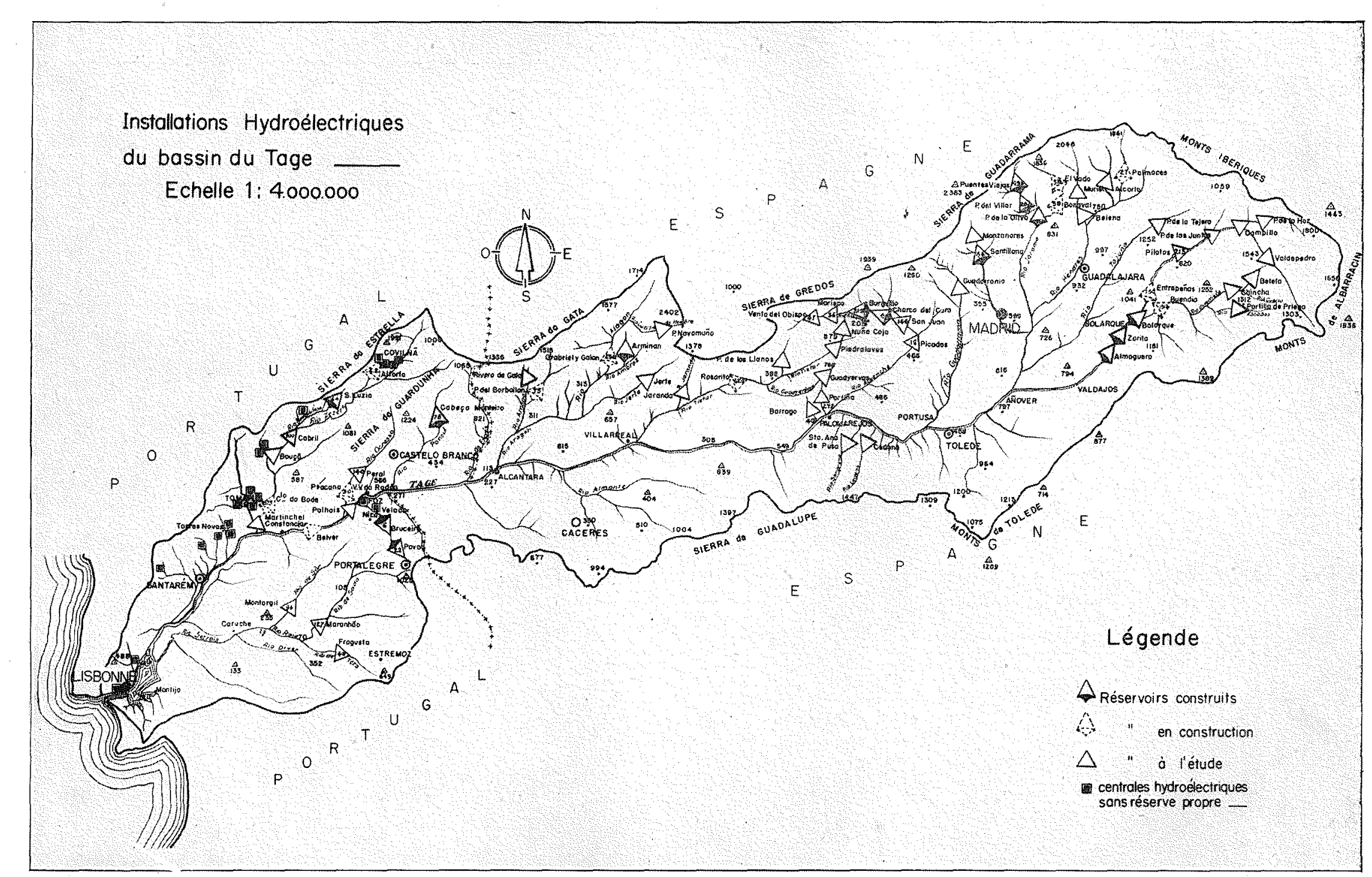


de l'ordre de 20 ou $30 \%$. Dans les années humides, la réduction obtenue sera plus faible, spécialement pour les crues de fin d'hiver ou de commencement du printemps.

Autre bénéfice certain : la grande réduction que subiront les débits solides du Tage à l'entrée au Portugal, ce qui présente une importance considérable pour la conservation du lit du lleuve et des champs marginaux sujets aux inon. dations. La régularisation du débit obtenue concourra à réduire le transport solide et une partie importante des matériaux sera arrètée par les retenues.

Les installations hydroelectriques existantes, en construction ou projetées dans le bassin portugais du Tage sont indiguées sur la Fig. VI, tandis que le Tableau II donne les principales. caractéristiques des plus importantes. Les valeurs numériques relatives aux installations en construction et plus spécialement à celles qui s: trouvent encore dans la phase de projet ou d'études sont sujettes à modification. Pour ce qui touche à la production d'énergie, il n'a pas été possible d'obtenir pour toutes les installafions des données d'égale rigueur, déterminées suivant le mème critérium (année sèche, année sèche moyenne ou année moyenne) et qui permettent de faire la distinction entre l'énergie permanente et l'énergie temporaire. Pour cela, les valeur's indiquées représentent seulement des ordres de grandeur des productions globales et ne sont pas entièrement comparables entre elles. Pour les installations du Tage, on n'a pas tenu compte de la régularisation possible des débits obtenue grace aux ouvrages espagnols parce que, comme il a été dit p'us haut, elle n'est pas suscepible de prévisions sùres.

Il existe actuellement dans le bassin portugais du Tage 27 centrales hydroélectriques produisant annuellement 60 mil'ions de kwh environ, dont neuf produisent à elles seules 58 millions de kwh anmuels et bénéficient de la régularisation des débits procurée par 5 retenues d'une capacité utile totalisant 150 millions de $\mathrm{m}^{3}$.

Quatre installations se trouvent actuellement en cours de construction et produiront $540 \mathrm{mil}$ lions de kwh environ, deux d'entre elles -.... Castelo do Bode sur le Zézere et Pracana sur l'Ocreza - disposant de retenues d'une capacité utile respective de 875 et 90 millions de m3.

En cours de projet ou d'étude sont encore cinq installations hydroćlectriques et trois installalions hydroagricoles produisant accessoirement de l'énergie dont les retenues tota'isent $900 \mathrm{mil}$ lions de $\mathrm{m}^{3}$ environ de capacité utile et qui produiront quelque 600 millions de kwh.

TABLEAU !I

Aménagements hydroélectriques dans le bassin hydrographique du Tage au Portugal.

\begin{tabular}{|c|c|c|c|c|c|c|}
\hline COURS D'EAY & DESIGNATYON & $\begin{array}{l}\text { Surface } \\
\text { bassin } \\
\text { : Km2 }\end{array}$ & $\begin{array}{l}\text { Hauteur } \\
\text { dal barrage } \\
\text { m. }\end{array}$ & $\begin{array}{l}\text { Volume utite } \\
\text { de la retente } \\
\text { mil. m3 }\end{array}$ & $\begin{array}{c}\text { Puissance } \\
\text { de Centrale } \\
\text { KVA } \\
\text { KVA }\end{array}$ & $\begin{array}{c}\text { Energie } \\
\text { produite } \\
\text { millions de Kwh }\end{array}$ \\
\hline TEJO & PALHAIS $\ldots \ldots \ldots \ldots$ I & 59.500 & 24 & . & 55.000 & 180 \\
\hline$»$ & BELYVER $\ldots \ldots \ldots$ C & 61.000 & 16 & . & 40.500 & 130 \\
\hline PONSUL & CABECO MONTEIRO . E & 358 & 45 & 28 & 1.900 & 7 \\
\hline OCREZA & PERAL $\ldots \ldots \ldots \ldots$ & 965 & 70 & 140 & 18.400 & $29^{\circ}$ \\
\hline$\gg \ldots \ldots$ & PRACANA $\ldots \ldots \ldots C$ & 1.410 & 60 & 90 & 18.400 & 27 \\
\hline ZEZERE $\ldots$ & CABRIL $\ldots \ldots \ldots \ldots$ & 2.340 & 135 & 500 & 96.600 & 210 \\
\hline$\gg \quad \ldots \ldots \ldots$ & BOUGA $\ldots \ldots \ldots \ldots$ P & 2.530 & 65 & $\ldots$ & 69.000 & 130 \\
\hline 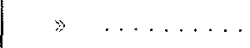 & CASTELO DO BODE . C C & 3.947 & 110 & 875 & 220.800 & 380 \\
\hline$» \ldots \ldots \ldots$ & CONSTANCIA $\ldots \ldots$ p & 4.020 & 12 & . & 11.000 & 30 \\
\hline ALFORTA . . . . & ALFORTA $\ldots \ldots \ldots$ C & 50 & 40 & 2 & 9.000 & 11 \\
\hline PAMPIHOSA $\ldots$ & SANTA LUZIA . . . E E & 50 & 75 & 44 & 22.000 & 30 \\
\hline NISA $\ldots \ldots$ & POVOA $\ldots \ldots \ldots \ldots$ E & 155 & 28 & 22 & 900 & 2 \\
\hline$\gg$ & BRUGEMA $\ldots \ldots$. E & 158 & 15 & 6 & 2.300 & 5 \\
\hline$\gg$ & VELADA $\ldots \ldots \ldots, \mathrm{E}$ & 209 & 8 & . & 5.500 & 8 \\
\hline$\gg \ldots$ & FOZ $\ldots \ldots \ldots \ldots \ldots$ E & 272 & 12 & . & 700 & . \\
\hline SEDA & MARANHAO $\ldots \ldots \ldots$ E & 2.282 & +7 & 127 & 6.300 & 13 \\
\hline SOR. & MONTARGIL . . . E E & 1.187 & 34 & 96 & 3.000 & 7 \\
\hline \multirow[t]{2}{*}{ TERA $\ldots \ldots \ldots$} & GRAGUSTA $\ldots \ldots \ldots$ E & & & 40 & & \\
\hline & . & & & 2.020 & 630.300 & 1.200 \\
\hline
\end{tabular}


Les installations figurant au tableau II sont groupées par bassins des principaux affluents du Tage sur le tableau III qui indique les surfaces tota'es des bassins versants et les surfaces dominées par les retenues et la valeur globale de leur capacité u'ile, la puissance des centrales et la production d'énergie.

Le tableau III nous montre que revient au Zézere la presque totalité de la production d'énergie $(88,8 \%)$ et que ses grandes reenues recouvrent environ $78 \%$ de la surface totale du bassin de cette rivière dans lesquels est comprise la zone de plus grande pluviosité. La régu- larisation obtenue par cette retenue se traduira par la restitution au Tage, d'un débit minimum de 60 à $80 \mathrm{~m}^{3}$ par seconde, qui augmentera en période d'étiage et atteindra à la fin de cette période des valeurs de l'ordre de 100 à $120 \mathrm{~ms} /$ sec. (suivant les caractéristiques hydrologiques des différentes années). Les retenues de l'Ocreza dominent la presque totalité $(98 \%)$ du bassin de cette rivière et l'on obtiendra avec elles un débit régularisé de $15 \mathrm{~m}^{3} / \mathrm{sec}$. qui à la fin de l'étiage attcindra approxima ivement $30 \mathrm{~m}^{3} / \mathrm{sec}$. (valeur dépendant des caractéristiques de l'année et de l'exploitation des centrales).

\section{TABLEAU III}

\begin{tabular}{|c|c|c|c|c|c|c|}
\hline \multirow{2}{*}{ AFFLCFNTS DU 'IAGF, } & \multirow{2}{*}{$\begin{array}{c}\text { Surfaces } \\
\text { totales des } \\
\text { bassins } \\
\text { Kmy }\end{array}$} & \multicolumn{2}{|c|}{$\begin{array}{c}\text { SURFACES RECOUVERTES } \\
\text { par les retenues }\end{array}$} & \multirow{2}{*}{$\begin{array}{c}\text { Volumes utiles } \\
\text { des retenues } \\
\text { Millions m3 }\end{array}$} & \multirow{2}{*}{$\begin{array}{l}\text { Puissance } \\
\text { des centrales } \\
\text { KVA }\end{array}$} & \multirow{2}{*}{$\begin{array}{l}\text { Production } \\
\text { d'énergie } \\
\text { millions de } K \text { wh }\end{array}$} \\
\hline & & $\mathrm{Km} 2$ & $\%$ & & & \\
\hline SORRAIA & $1 . \overline{6} 1 \overline{5}$ & 358 & 24 & 78 & 1.900 & 7 \\
\hline NISA $\ldots \ldots$ & 1.440 & 1.410 & 98 & 230 & 26.800 & 56 \\
\hline ZEZERE . & 5.023 & 3.947 & 78 & 1.421 & +28.400 & 791 \\
\hline OCREZA & 290 & 158 & 56 & 28 & $0+400$ & 16 \\
\hline PONSUL $\ldots \ldots \ldots \ldots$ & 6.270 & 3.700 & 59 & 263 & 9.350 & 20 \\
\hline
\end{tabular}

Les effets régularisateurs des autres retenues ont une inf'uence insignifiante sur le débit d'étiage du Tage, soit qu'elles n'aien: qu'une faible capacité ( $\mathrm{Ni}$ a) soit qu'elles soient destinées essentiellement à des fins hrdroagricoles (Ponsul et Sorraia). Les retenues du Zézere et de l'Ocreza avec de grandes capacités et recouvrant respectivement $78 \%\left(3947 \mathrm{~km}^{2}\right)$ et $98 \%(1.410$ $\mathrm{km}^{2}$ ) des surfaces de leurs bassins versants, absorbent les crues ordinaires et réduisent sensiblement les pointes des crues extraordinaires de ces aff'uents contribuant ainsi à une réduction appréciable des crues da Tage. La contribution apportée dans ce sens par les retenues du Ponsul et du Nisa ont peu d'importance, étant donnée la faible valeur de leur capacité et des surfaces par elles recouvertes. Quant aux retenues du Sorraia, elles n'auront d'effet favorable que sur leur propre bassin, car cette rivière se jette directement dans le large estuaire du Tage soumis à l'effet des marées.

Les installations en construction et projetées sur le Tage (Belver et Palhais) s'étendent sur toute la partie la plus inclinée du tronçon national de ce fleuve. A l'aval, existe encore une chute de l'ordre de 16 à $18 \mathrm{~m}$. que l'on pourra peutêtre utiliser pour produire de l'énergie, bien que dans cette partie de la vallée se présentent des sujétions très importantes d'ordre économique et social, comme il est dit plus haut. A l'amont restent seuls disponibles, à des fins hydroélectriques, les $28 \mathrm{~m}$. de chute du troncon in'ernational qui ne pourront être utilisés que moyennant un accord avec l'Espagne.

En plus des installations hydroélectriques in. diquées, quelques-unes pourront encore être réalisées dans des conditions que l'on présume? économiques sur les affluents de rive droite du Tage. On ne dispose pourtant pas encore d'assez de données pour se faire une idée suffisamment approchée de l'importance de ces installations dont les possibilités de production d'énergie doivent être de l'ordre de la centaine de millions de kwh.

L'augmentation des débits d'étiage et la réduction des débits de crues résultant de la régularisation effectuée par les retenues et la diminution des transports solides amélioreront les conditions de navigabilité du Tage, lesquelles seront encore rendues plus faciles dans les tronçons les plus inclinés de ce fleure, par les barrages établis dans sa vallée et munis d'écluses. Une fois réalisées les installations indiquées et après quelques travanx dans le lit du Tage, celui-ci deviendra navigable jusqu'à la frontière. 


\section{CONCLUSIONS}

1" -.. Le bassin hydrographique du 'Tage joue un ròle important dans la Péninsule Ibérique é constitue un facteur économique considérable dans l'ensemble de la Péninsule, tant par l'étendue des terres qui pourront être irriguées par ses caux, que par la grande quantité d'énergie que ses eaux peuvent produire ou encore par les possibilités de navigation dans son cours inférieur et dans son affluent Sorraia.

2" - Les ressources hydrauliques du Tage sont relativement modestes comparées à celles des rivières du Nord-Onest de la Péninsule, mais plus abondantes que celles des autres rivières a l'Est ou au Sud (par exemple, le Guadiana).

$3^{\circ}$ - Les centrales alimentées par les eaux du bassin hydrographique du Tage, existantes, en construction ou en projet, fourniront quelque 700 millions de $k w h$ en Espagne et 1.200 millions de kwh environ au Portugat. D'autres installations pourront se faire dans de bonnes conditions économiques produisant environ une centaine de millions de kwh au Portugal et quelques centaines de plus en Espagne.

4" - Les retenues des installations daus la zone espagnole du bassin du Tage avec une capacité totale utile de 3.600 millions de $\mathrm{m}^{3}$ donneront lieu, dans les prochaines années, à une augmentation du débit d'étiage du Tage, pouvant atteindre quelques dizaines de $m^{3}$ par seconde; ce bénéfice ira en diminuant avec l'intensification des irrigations en Espagne qui, dans un futur plus ot moins lointain pourront, peut-être, absorber presque complètement le débit d'étiage du Tage.

Les retenues des installations du Ziézere el de l'Ocreza fourniront au Tage des débits permanents minimum de l'ordre de 70 à $95 \mathrm{~m} 3$ see. qui augmentant au cours de l'étiage attẹindront it la fin de celui-ci des valeurs de l'ordre de 120 a $150 \mathrm{~m} 3 / \mathrm{sec}$.

$5^{\circ}$ - L'effet régularisateur des relenues espagnoles se traduira par une reduction de l'ordre de 20 à $30 \%$ des débits de crues à l'entrée au Portugal. Les retenues sur les affluents portugais, notamment sur le Zézere et l'Ocrezal, absorberont les crues ordinaires respectives de ces affuents et réduiront sensiblement les pointes des crues extraordinaires. Par suite, les crues du Tage subiront une réduction considérable, réduction qui sera plus faible en ammé humide, plus particulierement pour les crues do fin d'hiver ou de début de printemps.

$6^{\prime \prime-. . . ~ L a ~ r e ́ g u l a r i s a t i o n ~ d e s ~ d e ́ b i t s ~ o b t e n u e ~}$ concourra à réduire les débits solides dans le bassin du Tage et une grande partie des matériaux transportés sera arretée par les retentes. ce qui présente une importance considérable pour la conservation du lit du Tage et des champs marginaux sujets aux inondations.

$7^{\circ}$ - Une fois réalisées les installations hydróelectriques et après quelques travaux dans le lit du Tage, celui-ci deviendra navigable jusqu'à la frontière.

$8^{\circ}$-.. L'utilisation des ressources hydroćlectriques du bassin hydrographique du Tago pourra amener, pour le Ribatejo, les avantages suivants :

- énergie électrique abondante et à un prix qui permette une large utilisation, d'où résultera certainement un développement de l'industrie.

- une augmentation considérable des débits d'étiage du Tage, ce qui permettra de disposer d'eau pour irriguer de nombreux milliers d'hectares.

- une réduction appréciable des débits des crues et du transport solide du lleuve.

- une voie fluviale facilement navigable. 